Acta Universitatis Wratislaviensis No 3742

Studia Linguistica XXXV Wrocław 2016

DOI: $10.19195 / 0137-1169.35 .6$

JUDITH KREUZ

Pädagogische Hochschule Zug, Schweiz

VERA MUNDWILER

Universität Basel, Schweiz

\title{
„verbAndskasten !MÜS!sen wir haben“. Zum argumentativen Potenzial von Prosodie am Beispiel von Einigungsdiskussionen bei Grundschulkindern
}

\section{Einleitung ${ }^{1}$}

An Diskussionen teilnehmen zu können, die Argumente anderer zu verstehen und mit eigenen Argumenten darauf zu reagieren, ist zentraler Bestandteil des gesellschaftlichen Diskurses und der Aushandlung interpersonaler Beziehungen (Konfliktbearbeitung). Die Förderung des Erwerbs argumentativer Kompetenzen ist eng gekoppelt an den schulischen Bildungsauftrag und entsprechende Maßnahmen sind bereits ab dem Vorschulalter möglich (vgl. z.B. Kurtenbach et al. 2013). Insbesondere mündliches Argumentieren ist gemäß neueren Erkenntnissen für das schulische Lernen bzw. für den schulischen Erfolg generell wichtig und beeinflusst es positiv (vgl. z.B. Grundler/Vogt 2009).

Während es mittlerweile einige Studien zum mündlichen Argumentieren im Vorschulalter oder in der Sekundarstufe I und II gibt (vgl. z.B. Goetz/Shatz 1999, Felton/Kuhn 2001, Komor 2010, Blum-Kulka 2010, Grundler 2011, Zadunaisky Ehrlich/Blum-Kulka 2014, Krelle 2014, Arendt 2015), stellt v.a. die Erforschung von ungesteuerten Peer-Gesprächen unter Grundschulkindern hinsichtlich der Argumentationskompetenz ein Desideratum dar. Das vom Schweizerischen

\footnotetext{
${ }^{1}$ Wir bedanken uns bei Martin Luginbühl und Stefan Hauser sowie bei den HerausgeberInnen dieser Ausgabe für ihre Anmerkungen zu einer früheren Version dieses Artikels.
} 
Nationalfonds (SNF) geförderte, aktuell laufende Projekt „Argumentative Gesprächskompetenz in der Schule: Kontexte, Anforderungen, Erwerbsverläufe“2 nimmt dieses Desiderat auf und verfolgt das Ziel, Grundlagenwissen auch für anwendungsorientierte Fragestellungen zu liefern, insbesondere für die Förderung von Gesprächskompetenz im Schulunterricht in der Primarschulstufe.

Bei den hier vorgestellten argumentativen Gesprächen handelt es sich um Einigungsdiskussionen unter Grundschulkindern. In derartigen Diskussionen nehmen die GesprächspartnerInnen noch keine feste Position gegenüber der Problemstellung ein, sondern generieren diese im Gespräch selbst, indem sie verschiedene Lösungsmöglichkeiten einander gegenüberstellen (vgl. auch Grundler 2011:45) und diesbezüglich Argumente austauschen. Begründungshandlungen werden generell als Kernaufgabe des Argumentierens verstanden (vgl. Heller 2012), jedoch zeigt sich in unseren Analysen, dass die gemeinsame Überzeugungsarbeit nicht nur rein verbal-argumentativ geschieht, sondern in hohem Maße auch durch prosodische Sprechausdrucksmuster (vgl. stellvertretend z.B. Bose 2001, 2003:29), die als Begründung funktionalisiert werden (vgl. z.B. Müller 1999) oder ein wenig elaboriertes Argument in seiner Überzeugungskraft unterstützen. Sprechausdrucksmuster beschreiben Sprechausdrucksweisen, die sich regelmäßig wiederholen und geordnet erscheinen, also offensichtlich durch „soziale Prägungen" (Geißner 1984:25) anhand von Musterwissen der Kommunizierenden produziert (und rezipiert) werden (vgl. Gutenberg 1998:25). Die den Mustern zugrundeliegenden sprecherischen Merkmale sind beispielsweise Stimmhöhe, Lautheit, Stimmklang und Sprechgeschwindigkeit sowie Merkmalskomplexe wie Akzentuierung, Gliederung, Rhythmus etc. Diese sprecherischen Ausdrucksformen sind an sprachliche Formulierungen gebunden und werden einerseits zwar situations- und stimmungsabhängig konventionalisiert gestaltet, andererseits aber individuell ausgeführt (vgl. Krech et al. 1991). In den vorliegenden Daten lassen sich phonetische Markierungen identifizieren, die hochgradig persuasiv eingesetzt werden, wenn z.B. Verschiebungen des normativen Satzakzents auf Modalverben erfolgen, wodurch die Aussage erst ihren Status als Begründung gewinnt. Auch die Etablierung und Unterstützung epistemischer Autoritäten, denen im argumentativen Aushandlungsprozess eine zentrale Rolle zukommt, kann durch prosodische Mittel erfolgen. So bietet die Prosodie als Teilbereich der Interaktion den Teilnehmenden die Möglichkeit, sich zu positionieren und damit ihre kommunikativen Aktivitäten zu kontextualisieren und sprachlich zu markieren ${ }^{3}$ - und

2 Das Projekt wird unter der Leitung von Prof. Dr. Brigit Eriksson-Hotz (Pädagogische Hochschule Zug) und Prof. Dr. Martin Luginbühl (Universität Basel) durchgeführt; Laufdauer 2014-2017.

3 Gemäß Quasthoff und Krah (2012:116) verstehen wir Kontextualisierung als die Einpassung einer komplexen (argumentativen) Einheit in einen größeren Kontext, Vertextung als die der Gattung (hier Argumentieren) entsprechende sequenzielle Strukturierung und Markierung als sprachliches Anzeigen kontextueller und struktureller Bezüge. 
dies stets in direkter Beziehung zu den anderen Gesprächsteilnehmenden und deren Äußerungen (vgl. Szczepek Reed 2012).

Die Prosodie kann in der Interaktion als Ressource für die gemeinsame Bedeutungsherstellung genutzt werden und verweist demnach auf eine zusätzliche interaktionale Realität. Prosodische Mittel bieten damit ein großes Potenzial für erfolgreiche Argumentationsstrategien, deren Akzeptanz jedoch einer ständigen Aushandlung der Gesprächsteilnehmenden unterliegt. In diesem Beitrag zeigen wir, welchen Einfluss Prosodie auf die Ratifizierung und Akzeptanz von Argumenten und damit auf den Erfolg des Einigungsprozesses haben kann.

\section{Daten und Methoden}

Unser Korpus besteht aus 180 videografierten Kleingruppendiskussionen von jeweils vier zufällig zusammengestellten GrundschülerInnen einer Klasse (entweder der 2., 4. oder 6. Klasse mit entsprechend 7- bis 14-jährigen Kindern). Die Daten wurden im Zeitraum von Januar 2015 bis Januar 2016 an verschiedenen Primarschulen der deutschsprachigen Schweiz erhoben. Eine breite Streuung von ländlichen und urbanen Gegenden sowie unterschiedlichen sozialen Schichten ist gegeben.

Ausgangspunkt der Kleingruppendiskussion ist eine problemorientierte Fragestellung, die die SchülerInnen ohne inhaltliche Vorbereitung und ohne die Anwesenheit Erwachsener bearbeiten. Bei den Aufgabenstellungen handelt es sich um potenziell strittige Fragen, da mehrere Optionen möglich sind und die Kinder sich als Gruppe für eine Lösung entscheiden müssen. Die hier vorgestellten Daten beruhen auf dem Setting Robinson-Crusoe (vgl. ähnliches Aufgabendesign z.B. in Schilcher 2000), bei dem sich die SchülerInnen auf drei Gegenstände aus zwölf vorgegebenen einigen müssen, die sie nach einem Schiffsunglück vom Meeresgrund heraufholen können, um auf einer einsamen Insel zu überleben. Ebenso videografisch erfasst wurden die metareflexiven Nachgespräche mit der Versuchsleiterin.

Während es sich bei den Daten grundsätzlich um elizitierte Gespräche handelt und entsprechend die thematische Rahmung (einsame Insel) und das Ziel der Diskussion (Einigung) vorgegeben sind, müssen sich die Kinder nach der Instruktion dennoch selbst organisieren und eigenständig das Gespräch prozessieren. Da zudem keine Rollen zugewiesen werden und demnach die Steuerung und die lokale sowie globale Gesprächsorganisation den Kindern überlassen werden, sprechen wir von ungesteuerten Peer-Gesprächen.

Die aufgezeichneten Gespräche wurden unter Anwendung des gesprächsanalytischen Transkriptionssystems GAT 2 (Selting et al. 2009) zunächst transkribiert. Neben den verbalen Daten wurden auch die non- und paraverbalen Daten verschriftlicht, soweit sie im Sinne der Gesprächsanalyse als relevant gesetzt wer- 
den bzw. dem Verständnis dienen. Ausgewählte Beispiele wurden zudem mithilfe des Analyseprogramms PRAAT akustischen Analyseverfahren unterzogen (z.B. zur Bestimmung von Tonhöhenverläufen und Lautstärke), um die transkriptbasierenden Ergebnisse zu stützen.

Die Auswertung der videografierten Gespräche verortet sich in der ethnomethodologischen Gesprächsanalyse (vgl. z.B. Deppermann 2008). Hierzu wird grundsätzlich datengeleitet und induktiv vorgegangen (bottom up), indem keine a priori festgelegten Analyseschemata zur Anwendung kommen, sondern die Daten sequenzanalytisch und ergebnisoffen - allerdings mit Rückgriff auf die entsprechende Forschungsliteratur ${ }^{4}$ - betrachtet werden.

\section{Mündliches Argumentieren}

Das hier beschriebene Projekt schließt methodisch und thematisch weitgehend an einschlägige, gesprächsanalytische Studien von z.B. Spranz-Fogasy (2005), Grundler (2011) und Heller (2012) an. Somit wird hier deren grundsätzliche Auffassung von Argumentieren übernommen. Sie beschreiben Argumentieren als sprachliches Handeln, bei dem zwei oder mehr Personen gemeinsam ein im Gespräch als strittig markiertes Thema durch aufeinander bezogene sprachliche und nichtsprachliche Handlungen mündlich etablieren und argumentativ bearbeiten, also Positionen unter Rückgriff auf Begründungen und Plausibilisierungen erläutern und stützen (vgl. auch Andrews 2005:110, Vogt 2002:29f., Spranz-Fogasy 2003:31, 2005:146, Heller 2012). Dabei muss es sich nicht zwangsläufig um etwas Strittiges handeln, sondern es kann auch ein Abgleich von verschiedenen Perspektiven, ohne dass zwingend gegenteilige Meinungen verhandelt werden, im Mittelpunkt der Argumentation stehen (vgl. „Problematisierung“ bei Grundler 2011:114). Argumentieren ist dementsprechend ein global dimensioniertes, sequentiell strukturiertes und interaktiv hervorgebrachtes Problemlösungsverfahren zur begründeten und plausiblen Bearbeitung von differierenden Geltungsansprüchen (vgl. z.B. Quasthoff/Krah 2012, Heller 2012, Morek 2015).

So wird im Rahmen dieses Projekts einem erweiterten Argumentationsverständnis ${ }^{5}$ insofern Rechnung getragen, als dass für Begründungshandlungen auch weitere Diskursmuster wie Erklärungen, Erzählungen, Belehrungen und Mittel wie Sprechausdrucksmuster, bspw. prosodische Markierungen oder Modalisierungen berücksichtigt werden. Sowohl die verschiedenen argumentativ funktionalisierten Diskursmuster als auch die genannten persuasiven Mittel lassen sich zwar nicht den formal-logischen Argumenten i.e.S. als These und Begründung

${ }^{4}$ Deppermann (2006:17ff.) und Schwarze (2010:121ff.) verweisen auf die Problematik, dass unweigerlich auch voranalytische Annahmen zum Konzept des Argumentierens getroffen werden, die demnach nicht rein deskriptiv, sondern auch normativ geprägt sind.

${ }^{5} \mathrm{Zu}$ diesem erweiterten Begriff s. auch Grundler/Vogt 2009. 
(vgl. auch die pragma-dialektischen Ansätze nach v. Eemeren/Grootendorst 2004) oder expliziten Argumenten/Argumentationen ${ }^{6}$ zuordnen, jedoch können sie persuasiven Status erhalten und als Argument funktionalisiert werden. Es zeigt sich in den Daten immer wieder, dass eine Analyse von primär argumentativen Gesprächen ohne dieses weite Verständnis von Argumentieren kaum möglich ist, da sonst die Vielschichtigkeit von (gemeinsam) mündlich hervorgebrachten Äußerungen mit Begründungsstatus in einem Einigungsprozess unberücksichtigt bleibt (vgl. auch Studien zum mündlichen Argumentieren von z.B. Komor 2010, Morek 2015a, Arendt 2014 und 2015).

So beobachten wir in unseren Gesprächsdaten innerhalb der lokal eingebetteten Diskurseinheit Argumentieren ${ }^{7}$, dass die SchülerInnen nicht nur auf explizite Begründungshandlungen i.e.S. zurückgreifen, sondern dass sie sich vielfältiger persuasiver Strategien (z.B. prosodische Markierung von Behauptungen, Insistieren, Modalisierungen, Vorführen als Autorität) bedienen, um ihre Meinung in die Debatte einzubringen bzw. zu einem Konsens zu gelangen. Hinsichtlich des kindlichen spontanen Argumentierens kann an den Daten jedoch nur schwer rekonstruiert werden, was zur Kernaufgabe Begründen zählt, da die Argumente oft implizit bleiben. Es wird deswegen notwendig, ein geeignetes Konstrukt zur Identifikation von Argumenten aus den Daten heraus zu generieren. Dazu wird die Dichotomie von implizitem und explizitem Argumentieren aufgebrochen und stattdessen anhand eines Kontinuums von mehr oder weniger expliziten Handlungen beschrieben, über das die folgende Abbildung 1 modellhaft Aufschluss gibt:

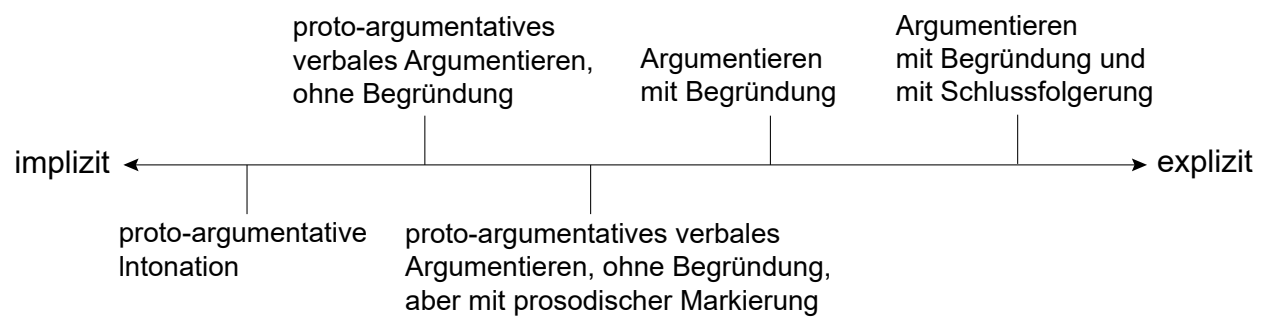

Abb. 1: Explizitheit argumentativer Handlungen

\footnotetext{
${ }^{6}$ Ein Argument gilt i.d.R. dann als explizit, wenn auf der sprachlichen Oberfläche entsprechende Begründungszusammenhänge (durch weil, denn, deshalb etc.) markiert werden (vgl. z.B. Deppermann 2006:18). Eine explizite Argumentation ist in der Rückschau ein erfassbarer ,vollständiger ${ }^{*}$ „Prämissen-Konklusions-Komplex“ (Deppermann 2006:14) mit den typischen Bestandteilen wie Behauptung/These, Begründung, Schlussregel (vgl. stellvertretend für klassische rhetorische Ansätze z.B. Kopperschmidt 2000).

${ }^{7}$ Vgl. globale Aufgaben/Jobs als definierende Bestandteile für das Argumentieren: Dissens herstellen/Problematisieren, Begründungspflicht etablieren, Kernaufgabe Begründen, Abschließen, Überleiten (Heller 2012:68ff., vgl. auch Hausendorf/Quasthoff 2005).
} 
Argumentationen eröffnen durch ihre unterschiedliche Charakteristik, ihre unterschiedliche Ausprägung im aktuellen sprachlichen Vollzug und ihren im Gespräch ausgehandelten Status einen variablen Raum auf einem Kontinuum zwischen implizit und explizit. Als hochgradig implizit und in dieser Form nicht Bestandteil unseres Korpus sind beispielsweise solche frühkindlichen Handlungen einzuordnen, die lediglich durch eine proto-argumentative Intonationskontur als argumentative Handlungen interpretierbar werden ${ }^{8}$ (vgl. zusammenfassend Bose 2003:50ff., vgl. auch Leitão 2010, Vasconcelos 2013, Vasconcelos et al. 2015). Die nächsten beiden Zwischenstufen auf dem Kontinuum von implizitem zu explizitem Argumentieren (Behauptung und Behauptung mit prosodischer Markierung ${ }^{9}$ ) lassen sich in unserem Korpus insbesondere bei den 7- bis 8-Jährigen finden. In dieser Altersgruppe scheint das argumentative Mittel der prosodischen Markierung also noch zu einer häufigen Praktik zu gehören. Im weiteren Verlauf des Kontinuums werden diese Praktiken durch explizite Verbalisierungen von Begründungen abgelöst, wie sie bereits in den Diskussionen der vierten Klasse und verstärkt in der sechsten Klasse beobachtbar sind. Ob es eine Korrelation zwischen der kindlichen Entwicklung und dem Grad der Explizitheit von Argumentationen gibt, kann zum jetzigen Analysezeitpunkt noch nicht validiert werden, sondern muss durch weitere Datenanalysen einer Verifizierung unterzogen werden.

\section{Formen und Funktionen von Prosodie}

In Gesprächen sind die Teilnehmenden in viele verschiedene sprecherische Handlungen involviert und es ist unbestritten, dass die Prosodie für die Ausführung und Koordination kommunikativer interaktiver Handlungen eine zentrale Rolle spielt (z.B. House 2006, 2009).

Für die Definition des Begriffs „Prosodie“ bzw. synonymisch „Suprasegmentalia“ lehnen wir uns an die aktuelle sprechwissenschaftliche Auffassung an und verstehen darunter einen Komplex an Merkmalen der gesprochenen Sprache, der die Parameter Sprechmelodie, Lautheit, Dauer, Sprechgeschwindigkeit, Sprechspannung, Pausen, Stimmqualität und Stimmausdruck beinhaltet (vgl. Hirschfeld/Neuber 2010, Neuber 2001:99, 2002:51f.). Prosodische Mit-

\footnotetext{
${ }^{8}$ Unter proto-argumentativen Intonationskonturen sind Sprechausdrucksweisen zu verstehen, die beispielsweise auf der gegenseitigen Nachahmung von Ausdrucksbewegungen und -lauten beruhen, „intonatorische Leitmuster“ darstellen oder durch eine „,intono-syntaktische Strukturierung“ gekennzeichnet sind (ausführlich dazu Bose 2003:50ff.). Prosodisch fallen v.a. starke Melodiebewegungen auf.

9 Das Modell ließe sich neben der prosodischen Markierung, die lediglich eine persuasive Strategie darstellt, um weitere ergänzen (z.B. Modalisierungen), für die anhand der Daten zu prüfen wäre, welche Platzierung in Bezug auf deren argumentativen Status innerhalb des Kontinuums angemessen ist.
} 
tel werden einzeln oder in Kombination (z.B. Akzentuierung, Rhythmus) „,in free variation" (Szczepek Reed 2012:16) eingesetzt und stehen in einem flexiblen Zusammenhang mit diskursiven Funktionen (vgl. Szczepek Reed 2004, Kaimaki 2011). Prosodische Markierungen dienen im Diskurs nicht nur dazu, individuelle Turns zu strukturieren, sondern auch Verbindungen zwischen den Äußerungen mehrerer Sprecher herzustellen (,cross-speaker synchronization“, Szczepek Reed 2012:14) und damit die gegenseitige Abhängigkeit unterschiedlicher Turns zu markieren. Prosodie bleibt somit nicht nur individuell, sondern wird auch kollaborativ von allen Gesprächsteilnehmenden verwendet, um das Gespräch gemeinsam zu strukturieren. Durch den melodischen Verlauf am Turnende und durch Pausensetzungen beispielsweise kann ausgehandelt werden, wie Rederechte (neu) verteilt werden. Wells (2010) zeigt in seiner Studie $\mathrm{zu}$,child-carer“-Konversationen auf, dass nicht nur die vom Kind spezifisch markierte Akzentuierung interaktiv bedeutsam ist, sondern dass es vielmehr auch dessen prosodische Wiederholung der mütterlichen Äußerung ist. Wells beobachtet weiterhin, dass SprecherInnen Bedeutung nicht durch prosodische Unterschiede herstellen, sondern eher Gebrauch von prosodischer Anpassung machen („tonal repetition“, Wells 2010:245).

Sowohl ihre (sprecherspezifische) Vielfalt, ihr Zusammenspiel mit der verbalen Sprache ${ }^{10}$ als auch ihre starke Kontextualität machen sie zu geeigneten Mitteln, um auch für argumentative Zwecke funktional sein zu können ${ }^{11}$ (vgl. Hirschfeld/Stock 2013:40):

- Durch ihre kommunikative Funktion können wichtige Informationen hervorgehoben und verdeutlicht sowie die Aufmerksamkeits- und Verstehensprozesse des/der Hörenden gesteuert werden. So können bereits einzelne Wörter prosodisch markiert werden, um innerhalb einer Diskussion persuasive Funktion zu erlangen und die Intention des/der Sprechenden anzuzeigen.

- Die gesprächsorganisierende Funktion bezieht sich auf die Gestaltung von Turns, Turnübernahmen und die Turnfortsetzung. Hierdurch kann bspw. das Rederecht (floor) ausgehandelt werden, was gerade in Diskussionen mit kompetitivem Charakter bedeutsam ist. In den Daten lässt sich beobachten, dass durch Insistieren bzw. durch non-lokale prosodisch markierte Wiederholungen der eigenen Meinung eine weitaus größere Chance besteht, dass diese Position argumentativ ausgehandelt und schlussendlich von allen akzeptiert bzw. zum Konsens wird. Gesprächsorganisierende Funktionen sind relevant, wenn beispielsweise

10 Die verbale Sprache meint in Abgrenzung zur paraverbalen Sprache die reine Silben- bzw. Wortebene. Diese wird in der gesprochenen Sprache jedoch immer von prosodischen Mitteln überformt. 11 Es muss jedoch berücksichtigt werden, dass diese vielfältigen Funktionen und Mittel der Prosodie oft gleichzeitig und kontextvariabel im Gespräch auftauchen. So können spezifische prosodische Phänomene nicht mit individuellen linguistischen Funktionen eindeutig in Verbindung gebracht werden (vgl. Szczepek Reed 2012). 
die individuelle Beitragslänge und die Sprechersymmetrie zur Etablierung eines/ einer informellen Gesprächsleitenden bzw. zur Hervorhebung einer epistemischen Autorität genutzt werden.

- Ebenso erfüllt auch die dritte Funktion - die expressive oder affektive Funktion - einen bedeutenden Zweck in Diskussionen, denn hierdurch können emotionale und modalisierte Sprechweisen markiert werden. „Prosody is used as a cue for the display of attitude, affect and stance" (Szczepek Reed 2012:17, vgl. auch Günthner 1996 und 1997, Müller 1996, Selting 1996, Freese/Maynard 1998, Kern 2010). Verbale und auch nonverbale Mittel werden hier in besonderem Maße durch prosodische Mittel begleitet. Diese Funktion ist für das Argumentieren i.S. von Streit, aber auch als Deeskalierungstaktik relevant.

Die einzelnen Funktionen werden durch ihre spezifische prosodische Markierung an der Oberfläche wahrnehmbar. Die Markierungen werden in den vielfältigen Gesprächskontexten häufig (unbewusst) ${ }^{12}$ adäquat eingesetzt; das muss aber nicht immer der Fall sein. So kann der Sprechausdruck beispielsweise nicht dem aktuellen Gesprächsmodus entsprechen, unbeabsichtigte Wirkungen erzielen und dann von den GesprächspartnerInnen als inakzeptabel klassifiziert werden. Dieser Umstand macht für die Sprechenden Wissen erforderlich, wie und wann prosodische Markierungen eingesetzt werden können und wie sie vom Gegenüber vermutlich rezipiert werden - um zu überzeugen, argumentative Handlungen zweckmäßig zu steuern oder deeskalierend zu wirken (vgl. die drei Funktionen der Prosodie). Prosodische Markierungen als mögliche, sprachliche Formen zur Indizierung argumentativer Strukturen“ (Morek 2015:34) können daher auch als Kompetenzmerkmal für (argumentative) Gespräche aufgefasst werden (vgl. Quasthoff 2009). Markierung wird von diesen beiden Autorinnen zwar als Kompetenz beschrieben, die sich vorrangig auf die sprachlichlexikalische Ebene bezieht, aber durchaus auch die prosodische Ebene mit einschließen kann, wenn man von funktionalen Markierungen in ihrer Gesamtheit und einer gegenseitigen Interdependenz auf verbaler und paraverbaler Ebene ausgeht. Prosodische und nonverbale Markierung wird von Quasthoff (2009:94) jedoch als implizite Markierung verstanden und erst mit zunehmendem Alter markieren Kinder ihre Äußerungen bzw. ihre Äußerungsabsicht expliziter durch die Lexik (vgl. Quasthoff 2009:90f.). Dies wurde beispielsweise von Quasthoff (2009:91) in Bezug auf das Erzählen und von Morek (2012, 2015b) in Bezug auf das Erklären gezeigt.

12 Bose (2003:89) vermutet, dass Sprechausdrucksmuster den Sprechenden nicht bewusst sein müssen und sie spricht daher von ,intuitiv-implizite[m] Erfahrungswissen“. 
Für das prosodische Markieren mit argumentativer Funktion ist augenfällig, dass in unserem Korpus v.a. die jüngeren Kinder darauf zurückgreifen. Dies deckt sich mit Boses (2003:89) Feststellung, dass aufgrund der Basisfunktionen von Prosodie sowie entsprechenden Forschungsergebnissen zur frühen Eltern-Kind-Kommunikation erwartbar ist, dass ,,schon sehr kleine Kinder über eine Sprechausdruckskompetenz verfügen, die ihre Sprachkompetenz übersteigt" ${ }^{13}$ So bleiben prosodische Markierungen im Gegensatz zu lexikalischen Markierungen im Erwerbsverlauf eher eine implizite Ressource, da sie keine verfestigten bzw. starren Muster der Sprache sind, sondern situationsabhängig und individuell gebraucht werden. Sie sind ,immer nur im Zusammenspiel mit anderen verbalen und kinetischen Ressourcen in einem bestimmten sequentiellen Kontext interpretierbar“ (Kupetz 2014: 92, vgl. auch Couper- Kuhlen/Selting 1996, Selting 2013). Dennoch zeugt deren tatsächliche Verwendung sowohl im Gespräch im Allgemeinen als auch als argumentatives Mittel in Einigungsdiskussionen von einer sprech-sprachlichen Kompetenz.

\section{Argumentative Funktionalisierung von Prosodie}

Im Folgenden zeigen wir anhand von Beispielen aus dem Korpus, wie Grundschulkinder die Prosodie als persuasive Strategie nutzen.

\subsection{Markierung von impliziten Argumenten durch spezifische Akzentsetzung ${ }^{14}$}

Das erste Beispiel ${ }^{15}$ stammt aus einer zweiten Klasse. Im Vorfeld des gewählten Ausschnitts positioniert sich Renato ${ }^{16}$ (argumentativ) zu drei Gegenständen seiner Wahl. Diese werden von Edgardo abgelehnt, indem er seinerseits einen vierten Gegenstand, den Verbandskasten, unbegründet - lediglich durch Zeigegesten begleitend - vorschlägt (Zeile 001):

13 Gemäß Bose (2003:88f.) werden unter Sprechausdruckskompetenz ,überwiegend implizite Kenntnisse über und Fähigkeiten zur situations- und stimmungsabhängigen Variation von Sprechausdrucksweisen verstanden, die als Regulationsbasis für kommunikatives Handeln dienen“".

${ }^{14}$ Akzentuierte Silben werden im Gegensatz zu unbetonten Silben i.d.R. langsamer, lauter und höher gesprochen, zudem sind sie durch eine hohe Sprechspannung gekennzeichnet (vgl. stellvertretend z.B. Hirschfeld/Stock 2013:41).

15 Die Kinder sprechen i.d.R. eine dialektale, schweizerdeutsche Varietät, orientieren sich aber teilweise auch an der Schulsprache (Standarddeutsch). In den Transkripten finden sich jeweils fett markiert die originalen Äußerungen und interlinear die standardsprachlichen Übersetzungen.

16 Alle Gespräche wurden anonymisiert und es handelt sich bei den Namen entsprechend um Pseudonyme. 
Beispiel 1: Ro_K2_HZ_G5a (Nick, Marc, Edgardo, Renato), Min. 07:00-07:08

\begin{tabular}{|c|c|c|}
\hline 001 & EDG: & $\begin{array}{l}\text { abEr nÄI: dAs dA DAS dA:; ( (tippt auf das } \\
\text { Verbandszeug auf Marcs Blatt)) } \\
\text { aber nein das da das da }\end{array}$ \\
\hline 002 & NIC: & $\begin{array}{l}\text { dAs wÖMmer ja [hAA; ] ( (streckt die linke } \\
\text { Hand zu MARs Blatt aus)) } \\
\text { das wollen wir ja haben }\end{array}$ \\
\hline 003 & MAR: & $\begin{array}{l}\text { [ja (.) ] verbAnd }{ }^{\circ} \mathbf{h} \text { kAsten } \\
\text { ! Mü:S!sen wir hAben; } \\
\text { ja Verbandkasten müssen wir haben } \\
\text { ( (beugt sich leicht nach vorne und breitet } \\
\text { die Hände unterstreichend vor sich an der } \\
\text { Tischkante aus, lehnt sich wieder nach } \\
\text { hinten)) }\end{array}$ \\
\hline 004 & EDG: & $\begin{array}{l}\text { DAS (.) müe MüEmer haa; } \\
\text { das mü müssen wir haben }\end{array}$ \\
\hline
\end{tabular}

Nick und Marc stützen den Vorschlag, ebenso ohne verbale Begründung, aber durch auffällige prosodische Markierungen. Dabei fällt v.a. Marcs Äußerung in Zeile 003 auf: ,ja (.) verbAnd ${ }^{\circ} \mathrm{h}$ kAsten !MÜ:S!sen wir hAben;“. Auch auf lexikalischer Ebene ist interessant, wie Marc einen Wechsel von Nicks „wollen“ (Zeile 002) zum extrasubjektiven „müssen“ (Zeile 003) vornimmt und dadurch die argumentative Funktion seiner Aussage verstärkt. Edgardo stimmt in Zeile 004 explizit zu, indem er Marcs Äußerung sowohl verbal wiederholt als auch nach einer selbstinitiierten Selbstreparatur dessen prosodisches Muster, v.a. die Akzentsilbe, imitiert (,prosodical matching“, vgl. dazu z.B. Szczepek Reed 2012). Nick und der bis dahin oppositionelle Renato zeigen daraufhin ihre multimodale Ausrichtung an Marcs Beitrag und in einer späteren Sequenz wird der Verbandskasten zudem verbal ratifiziert. Ein gemeinsamer Konsens ist gefunden, auch ohne dass verbal eine Begründung geäußert wird. Die paraverbale Markierung reicht im vorliegenden Kontext aus, um den Vorschlag als Argument zu akzeptieren.

Ein zweites Beispiel verdeutlicht, wie durch Akzentuierung einem argumentativ wenig elaborierten Argument Bedeutung verliehen wird:

Beispiel 2: Ro_K2_HZ_G3a (Jonas, Nevio, Carlos, Samuel), Min. 03:13-03:45

\begin{tabular}{|c|c|c|}
\hline 001 & SAM: & $\begin{array}{l}\text { aso uf jede fall lüÜchtrakEte BRUUche mer; } \\
\text { also auf jeden Fall Leuchtrakete brauchen } \\
\text { wir }\end{array}$ \\
\hline 002 & JON : & JA, \\
\hline 003 & SAM: & $\begin{array}{l}\text { LüÜCHTrAkete das scho mol klAr; } \\
\text { Leuchtrakete das ist schon mal klar }\end{array}$ \\
\hline
\end{tabular}




\begin{tabular}{|c|c|c|}
\hline 004 & & ((Auslassung von 23.0 sek.)) \\
\hline 005 & SAM: & $\begin{array}{l}{ }^{\circ} \text { hh Aso (.) ich glaub lüÜchtrakEte dasch } \\
\text { jetz scho mol KLAR; } \\
\text { also ich glaub Leuchtrakete das ist jetzt } \\
\text { schon mal klar }\end{array}$ \\
\hline 006 & JON : & JA； \\
\hline 007 & NEV: & $\begin{array}{l}\text { jA (.) LÜÜCHTrakEte (-) Abgschlosse. } \\
\text { ja Leuchtrakete abgeschlossen }\end{array}$ \\
\hline
\end{tabular}

Die Aussage von Samuel „aso uf jede fall lÜÜchtrakEte BRUUche mer“ enthält die für unser Setting typische Wendung „brauchen wir“, für die analytisch unklar ist, ob sie als Argument i.e.S. gelten kann oder nicht. Da es in der Diskussion darum geht zu entscheiden, welche Gegenstände die Kinder auf einer einsamen Insel zum Überleben brauchen, scheint die Verwendung von brauchen als Reaktivierung des bereits etablierten common ground (vgl. z.B. Clark 1996) zu fungieren. Die tatsächliche Begründung bleibt somit häufig aus, da alle Kinder über die Ausgangslage Bescheid wissen. Das akzentuierte brauchen wird hier also verwendet, um auf die Begründbarkeit hinzuweisen, ohne aber explizite Begründungen verbalisieren zu müssen. So wird Samuels Äußerung ohne weitere explizite Begründung durch Jonas ratifiziert (Zeile 002, 006) und von Samuel wiederholt als Konsens eingestuft (mit einem Akzent auf „KLAR“, Zeile 003, 005). Für Samuel besteht also keine weitere Notwendigkeit, eine explizitere Begründung zu liefern - seine starke Akzentuierung ist für die Persuasion seiner Mitschüler ausreichend. Aber auch seine damit etablierte (epistemische) Autorität trägt zu einer persuasiven Wirkung bei. Die anderen Schüler scheinen nicht die Intention zu haben, auf ein solch stark markiertes Sprechausdrucksmuster zu widersprechen. So wird die Argumentation verbal abgeschlossen (Zeile 007).

\section{2. Überzeugen durch prosodische Markierung und Etablierung einer epistemischen Autorität}

Wie im obigen Beispiel bereits angedeutet wurde, nimmt in argumentativen Aushandlungen vielfach auch die epistemische Autorität eine zentrale Stellung für das Überzeugen ein. Es kann also auch das (teils prosodisch markierte) argumentative Verfahren der Überzeugung qua Autorität als argumentatives Mittel verstanden werden, nämlich, wenn informelle GruppenleiterInnen und epistemische Autoritäten durch prosodische Markierung etabliert bzw. gestützt werden.

Das folgende Beispiel ist dadurch charakterisiert, dass während des gesamten Gesprächs ein primärer Sprecher (Tom) das Rederecht innehat und neben einigen inhaltlichen Argumenten v.a. durch seinen autoritativen Sprechausdruck überzeugt, der sich insbesondere durch deutliches, gespanntes und stark akzentuiertes Spre- 
chen auszeichnet (Bose 2003:227). Im Datum wird ersichtlich, wie Tom prosodisch auffällig seine Äußerungen bzw. Widersprüche markiert und damit seine Begründungen unterstützt:

Beispiel 3: Ro_K4_HZ_G4b (Max, Jan, Liz, Tom), Min. 02:33-03:23

\begin{tabular}{|c|c|c|}
\hline 001 & TOM: & $\begin{array}{l}\text { ä2ä (.) näI (.) ebe NÖD; } \\
\text { ä ä nein eben nicht }\end{array}$ \\
\hline 002 & & $\begin{array}{l}{ }^{\circ} \mathbf{h} \text { me cha mit em SACKmässer? } \\
\text { man kann mit einem Sackmesser }\end{array}$ \\
\hline 003 & & $\begin{array}{l}\text { (--) chA mer gäg en: STÄI schloo? } \\
\text { kann man gegen einen Stein schlagen }\end{array}$ \\
\hline 04 & & $\begin{array}{l}\text { und dänn gits FuNke; } \\
\text { und dann gibt's Funken }\end{array}$ \\
\hline 05 & & $(1.6)$ \\
\hline 006 & JAN : & $\begin{array}{l}\text { Ah: oKEE; } \\
\text { ah okay }\end{array}$ \\
\hline 007 & LIZ: & $a h$ \\
\hline 008 & TOM: & $\begin{array}{l}\text { und dAs git Füür; } \\
\text { und das gibt Feuer }\end{array}$ \\
\hline 009 & & $\begin{array}{l}\text { Also bruucht me no s SACKmässer? } \\
\text { also braucht man noch das Sackmesser }\end{array}$ \\
\hline 010 & & $((\ldots))$ \\
\hline 011 & TOM: & $\begin{array}{l}\text { nä nÄI }{ }^{\circ} \text { h nÄI CHOCHtopf doch nöd; } \\
\text { nein nein nein Kochtopf doch nicht }\end{array}$ \\
\hline 012 & & $\begin{array}{l}{ }^{\circ} \mathrm{h} \text { das chA mer au irgend uf em ne (.) im ene } \\
\text { FLuss zueberÄIte; } \\
\text { das kann man auch irgend auf einem in einem } \\
\text { Fluss zubereiten }\end{array}$ \\
\hline 013 & & $\begin{array}{l}\text { =aber ! ICH! würdi vor allem zält oder } \\
\text { moskItonetz nee. } \\
\text { aber ich würde vor allem das Zelt oder } \\
\text { Moskitonetz nehmen }\end{array}$ \\
\hline 014 & & $((\ldots))$ \\
\hline 015 & TOM: & $\begin{array}{l}\text { okee ! ICH! sÄg; } \\
\text { okay ich sage }\end{array}$ \\
\hline 016 & & $\begin{array}{l}\text { moski äH verBANDSzüüg? } \\
\text { Moski äh Verbandszeug }\end{array}$ \\
\hline 017 & & $\begin{array}{l}\text { LüÜCHTrakete und sAckmässer. ((wendet sich } \\
\text { der Gruppe zu und verschränkt die Arme)) } \\
\text { Leuchtrakete und Sackmesser }\end{array}$ \\
\hline
\end{tabular}

Die Aushandlung findet schnell ein Ende, da ein offensichtlicher Entscheid gefallen ist, der von Tom festgelegt wurde; es erfolgt kein Widerspruch der Gruppe. 
Tom etabliert sich einerseits durch sein Wissen und seine Begründungen als epistemische Autorität, andererseits aber auch durch die starke Betonung seiner unbegründeten Behauptungen/Vorschläge durch die auffällig starke Markierung von ,!ICH!“ (Zeile 013, 015). Auch bei seinen einfachen unbegründeten Widersprüchen (,nÄI (.) ebe NÖD;“, Zeile 001), die durch eine langsame, akzentuierte Sprechweise und Pausensetzung sowie eine fallende Melodie am Turnende gekennzeichnet sind, wird seiner Äußerung und dem folgenden Erklärstück eine hohe Bedeutsamkeit verliehen bzw. der Aufmerksamkeitsfokus auf ihn als bereits etablierte epistemische Autorität aktualisiert.

\subsection{Ko-Konstruiertes Argumentieren}

Unsere dritte Beobachtung zielt auf die stark ko-konstruierte Aushandlung von Positionen, für die in enger syntaktischer, inhaltlich-argumentativer und prosodischer Abhängigkeit gemeinsam Argumente und Gegenargumente elaboriert werden (vgl. auch Hauser/Luginbühl 2015). Mit dem Fokus auf die Prosodie lässt sich häufig beobachten, wie prosodische Anpassungen zweiter SprecherInnen an die Vorgängeräußerungen (,prosodic matching“, z.B. Couper-Kuhlen 1996/2012, Tarplee 1996, Wells/Corin 2004, Szczepek Reed 2012) dazu dienen können, um beispielsweise affiliierende Allianzen zu bilden und Argumente gegenseitig persuasiv zu verstärken (s. dazu auch Szczepek 2000a/b). Diese prosodischen Anpassungen sind dabei auf verschiedenen Ebenen wie Tonhöhenverlauf, Lautstärke, Sprechgeschwindigkeit, Lautproduktion, Rhythmus etc. möglich (vgl. Auer/Couper-Kuhlen 1994, Kupetz 2014, Ogden 2006, Szczepek Reed 2006) und begleiten eine Bandbreite an kommunikativen Interaktionen (Szczepek Reed 2006). Die interaktive Herstellung von Gemeinsamkeit kann in Anlehnung an Glindemann (1987, vgl. auch Bose 2003:339) auch als „Sprechausdruckskonsonanz“ bezeichnet werden.

Folgendes Beispiel verdeutlicht eine ,prosodic orientation“ (Szczepek Reed 2006:12) mit der Unterform des „prosodic matching“17 (ebd.:19) zweier sprecherverschiedener Äußerungen aneinander:

Beispiel 4: Ro_K4_HZ_G1b(Lena, Julia, Tevin, Simon), Min. 03:02-03:06

$\begin{array}{lll}001 & \text { JUL: das ist wichtig aber dann können wir nur } \\ & \text { noch <<klagend> ETwas;> } \\ 002 & \text { SIM: } & \begin{array}{l}\text { FEUer fEUer fEUer, ( (zeigt aufs Blatt, dann } \\ \text { schweifende Handbewegung nach außen)) }\end{array} \\ & \text { jA FEUer aber (.) aber- ((verwirft die Hände } \\ & \text { in der Luft)) }\end{array}$

17 Weitere Unterformen sind „prosodic complementation“, „prosodic continuation“ und ,prosodic non-matching“" (Szczepek Reed 2012:19). 
Zunächst bezieht Simon Stellung und verweist auf die Notwendigkeit von Feuer („FEUer fEUer fEUer,“). Seine dreimalige Wiederholung des Lexems Feuer ist mit einer hohen Sprechspannung, starker Lautstärke und mehreren Akzenten markiert (vgl. zur affektiven Funktion von Akzenthäufungen auch Bose 2003:151). Er nimmt dadurch eindeutig, wenn auch spontan-assoziativ Stellung. Seine Meinung wird durch sein emphatisches Sprechausdrucksmuster argumentativ funktionalisiert und als hochgradig relevant markiert. Eine verbale Begründung bleibt vorerst aus, jedoch wird seine Äußerung nun angreifbar. Es ist Julia, die auch sogleich Dissens markiert: ,jA FEUer aber (.) aber-“ . Auf der lexikalischen Ebene wiederholt sie das problematische Element Feuer und markiert dadurch den strittigen Punkt (vgl. auch „Dissensformate“ bei Günthner 2000:219f. oder „Pseudodarstellung“ bei Reber 2009). Durch das Mittel des „format tying“ (Goodwin 2006:449) als Wiederverwendung, aber auch durch die Modifikation des kontextuellen Materials, kann Julia einen oppositionellen Zug konstruieren und eine Begründung einfordern. Sie passt sich auch in ihrem Aktivitätsmodus der Vorgängeräußerung an, denn ,participants design each instance of talk according to the locally emerging contingencies" (Szczepek 2012:18). Durch ihre ebenfalls hohe Sprechspannung, ihre starke Betonung und die erhöhte Laustärke zeigt sie an, die Aushandlung des aktuellen Themas und den Gesprächsmodus aufrechterhalten zu wollen. Ogden (2006) zeigt, dass sowohl Zustimmungen als auch Widersprüche in Abhängigkeit zur vorherigen Äußerung ,phonetically upgraded“ (Ogden 2006:1772) sein können. Sie enthalten „expanded pitch spans, more dynamic contours on accented syllables, higher pitch range, reduced speech rate and closer articulation" (ebd.:1762). In authentischen Gesprächsdaten wird immer wieder die große Rolle von Intonations- und Rhythmusübernahmen offensichtlich, die auch dazu dienen können, den Grad der phatischen Zuwendung anzuzeigen. Diese durch prosodische Entsprechungen (u.a.) zwischen zwei Äußerungen hervorgerufene intensivierte Form von Gemeinsamkeit bezeichnet Schwitalla (1993:79) als „kollektives Sprechen“ (Schwitalla 1993:74). Für Schwitalla ist „,g]roße prosodische Expressivität [...] eins der Mittel, mit denen man über eine kooperative Einstellung hinaus eine außergewöhnliche, exaltatorische Übereinstimmung herstellen kann" (ebd.:80). Im argumentativen Gespräch kann diese Anpassung, wie in obigem Beispiel, allerdings auch disaffiliierenden Charakter haben und oppositionelle Haltungen anzeigen. Weder Julia noch Simon explizieren sprachlich ihre opponierenden Positionen durch vollständige argumentative Strukturelemente, sondern fangen den fragmentarischen Charakter ihrer Argumentation durch prosodische Markierung sowie ihre prosodischen Imitationen ab, markieren damit aber dennoch eindeutig ihre inkompatiblen Sichtweisen/das Strittige für den Partner/die Partnerin. Die prosodischen „Abschattierungen“ (ebd.:80) tragen dazu bei, eine gemeinsame Stimmung herzustellen und den Handlungstyp des Argumentierens zu markieren und kontextadäquat einzubinden (Kontextualisierung), um so auf Grundlage eines gemeinsamen Gesprächsmodus zu agieren. Es 
wird deutlich, wie eng die Kinder an einem gemeinsamen Ziel durch Behaupten, aber auch Einwenden arbeiten. Gerade durch die Prosodie werden ,togetherness“ (Szczepek 2000b:12) und die gegenseitige Verständigung der Interagierenden offensichtlich. Die prosodische Imitation lässt sich als Kompetenz verstehen, die im Kontext solcher ,joint attentional frames“ (Tomasello 2003) aufkommt: die kommunikative Intentionen des Gegenübers zu verstehen, sich stark an dem Partner/der Partnerin zu orientieren (auch durch Hörverstehen), synchronisiert zu denken sowie die eigenen Äußerungen sprachlich-sprecherisch zu vertexten und zu markieren.

\section{Diskussion und Schlussfolgerung}

Argumentative Interaktionen zählen zu einem komplexen Handlungstyp, in dem viele verschiedene sprachliche und sprecherische Ressourcen simultan zum Tragen kommen, um interaktiv gemeinsam Bedeutung herzustellen sowie Gesprächsmodi und -ziele auszuhandeln. Interaktionale Prosodie ist eine dieser Ressourcen, die aber immer im Zusammenhang mit anderen interaktionalen Ressourcen und den sich laufend verändernden kontextuellen Faktoren gesehen werden muss. Einer einfachen Relation zwischen prosodischen Mitteln und konversationellen Aktivitäten muss in gesprächsanalytischen Prosodiestudien dieser Art daher mit Vorsicht begegnet werden. Prosodie kann nicht nur als monologisches Mittel charakterisiert werden, sondern schließt durch sein Auftreten bspw. im „prosodic matching“ die Dialogizität von Interaktionen mit ein. So ist eine fundamentale Charakteristik prosodischer Orientierung ,that next speakers display awareness of, and make relevant previous speakers' prosodic patterns“" (Szczepek Reed 2012:22). Wie wir anhand der Daten zeigen konnten, leisten prosodische Realisierungen einen Beitrag zur Interpretation von (proto-)argumentativen Handlungen insbesondere bei jüngeren Kindern. Sie nutzen Prosodie zur Persuasion und damit argumentativ als Markierung von impliziten Argumenten durch eine spezifische Akzentuierung (vgl. Beispiel 1), als Kompensation fehlender Begründungshandlungen (vgl. Beispiel 2), als persuasive Verstärkung der Argumentation (vgl. Beispiel 3) und als Markierung von argumentativen Zusammengehörigkeiten im kollaborativen Handlungsprozess bzw. als Markierung von kollaborativen Ergänzungen, Stützungen o.ä. zu bereits geäußerten Argumenten (vgl. Beispiel 4). Weiterhin gelingt es den Kindern durch eine gegenseitige prosodische Orientierung (v.a. in Bezug auf die Aushandlung von Turnübernahmen), ihre epistemische Autorität zu etablieren bzw. zu unterstützen, auf Begründbarkeit hinzuweisen, ohne aber Begründungen verbalisieren zu müssen und Dissens durch prosodische Anpaßung an Vorgängeräußerungen (,prosodic matching", vgl. z.B. Couper-Kuhlen 1996 und 2012) zu kontextualisieren. Prosodische Akzentuierung und die Verwendung bestimmter Sprechausdrucksmuster kann daher als implizite Markierungskompetenz angesehen werden. 
Um erwerbsspezifische Fragen der mündlichen Argumentationskompetenz beantworten zu können, ist es daher notwendig, einen erweiterten Argumentationsbegriff zu verwenden, damit ein breites Spektrum an persuasiven Mitteln berücksichtigt werden kann. Für den didaktischen Zugang zum Erwerb von argumentativen Fertigkeiten bedeutet das, dass die schulische Normvorstellung in Bezug auf (konstruiertes) „kompetentes“ Argumentieren geöffnet werden könnte und zu Gunsten eines flexiblen und prüfendes Umgangs an eine stärker gebrauchsnormierte Lernumgebung und entsprechend an eine implizite funktionale soziale Praxis angelehnt werden dürfte.

\section{Literatur}

ANDREWS Richard, 2005, Models of Argumentation in Educational Discourse, in: Interdisciplinary Journal for the Study of Discourse 25(1), S. 107-112.

ARENDT Birte, 2014, Konfliktbearbeitung von Kindergartenkindern - verbale resp. argumentative und nonverbale Muster, in: Mémoires de la Société Néophilologique de Helsinki, S. 21-34.

ARENDT Birte, 2015, Kindergartenkinder argumentieren - Peer-Gespräche als Erwerbskontext, in: Arendt B./Heller V./Krah A. (Hrsg.), Themenheft «Kinder argumentieren. Interaktive Erwerbskontexte und -mechanismen», Mitteilungen des Deutschen Germanistenverbandes, S. 21-33.

AUER Peter / COUPER-Kuhlen Elizabeth, 1994, Rhythmus und Tempo in konversationeller Alltagssprache, in: Schlieben-Lange B. (Hrsg.), Rhythmus: Zeitschrift für Literaturwissenschaft und Linguistik 24(96), S. $78-106$.

BLuM-KulKa Shoshana / SNOw Catherine, 2004, Introduction: The Potential of Peer Talk, in: Discourse Studies 6(3), S. 291-306.

BLUM-KUlKa Shoshana, 2010, Explanations in Naturally Occuring Peer Talk, in: First Language 30 , S. $440-460$.

Bose Ines, 2001, Methoden der Sprechausdrucksbeschreibung am Beispiel kindlicher Spielkommunikation, in: Gesprächsforschung - Online-Zeitschrift zur verbalen Interaktion 2, S. 262-303.

Bose Ines, 2003, Dóch da sín ja' nur mûster. Kindlicher Sprechausdruck im sozialen Rollenspiel, Frankfurt a. M.

ClaRK Herbert H., 1996, Using Language, Cambridge.

COUPER-KuHLEN Elizabeth, 1996, The Prosody of Repetition: On Quoting and Mimicry, in: CouperKuhlen E./Selting M. (Hrsg.), Prosody in Conversation: Interactional Studies, Cambridge, S. $366-405$.

COUPER-KUHLEN Elizabeth, 2012, Exploring Affiliation in the Reception of Conversational Complaint Stories, in: Peräkylä A./Sorjonen M. (Hrsg.), Emotion in Interaction, Oxford, S. $113-146$.

COUPER-KuHLEN Elizabeth / SELTING Margret, 1996, Towards an Interactional Perspective on Prosody and a Prosodic Perspective on Interaction, in: Couper-Kuhlen E./Selting M. (Hrsg.), Prosody in Conversation, Interactional Studies, Cambridge, S. 11-56.

DEPPERMANN Arnulf, 2006, Desiderata einer gesprächsanalytischen Argumentationsforschung, in: Deppermann A./Hartung M. (Hrsg.), Argumentieren in Gesprächen. Gesprächsanalytische Studien, 2. Aufl., Tübingen, S. 10-26.

DEPPERMANN Arnulf, 2008, Gespräche analysieren. Eine Einführung in konversationsanalytische Methoden, 4. Aufl., Wiesbaden. 
DunN Judy / MunN Penny, 1987, Development of Justification in Disputes with Mother and Sibling, Developmental Psychology 23, S. 791-798.

FeLTON Mark / KUHN Deanna, 2001, The Development of Argumentive Discourse Skills, in: Discourse Processes 32(2\&3), S. $135-153$.

FreESE Jeremy / MAYNARD Douglass W., 1998, Prosodic Features of Bad News and Good News in Conversation, Language in Society 27, S. 195-220.

GEIßNER Hellmut, 1984, Über Hörmuster. Gerold Ungeheuer zum Gedenken, in: Gutenberg N. (Hrsg.), Hören und Beurteilen: Gegenstand und Methode der Sprechwissenschaft, Sprecherziehung, Phonetik, Linguistik und Literaturwissenschaft, Sprache und Sprechen 2, S. 13-56.

GLINDEMANN Ralf, 1987, Zusammensprechen in Gesprächen. Aspekte einer konsonanz-theoretischen Pragmatik, Tübingen.

Goetz Peggy J. / Shatz Marilyn, 1999, When and How Peers Give Reasons: Justifications in the Talk of Middle School Children, in: Journal of Child Language 26(3), S. 721 - 748.

Goetz Peggy J., 2010, The Development of Verbal Justifications in the Conversations of Preschool Children and Adults, in: First Language 30(3-4), S. 403-420.

GoodwIN Charles, 2006, Retrospective and Prospective Orientation in the Construction of Argumentative Moves, in: Text and Talk 26, S. $441-460$.

GRUNDLER Elke / VOGT Rüdiger, 2009, Diskutieren und Debattieren. Argumentieren in der Schule, in: Becker-Mrotzek M. (Hrsg.), Mündliche Kommunikation und Gesprächsdidaktik, Baltmannsweiler, S. 487-511.

GÜNTHNER Susanne, 1996, The Prosodic Contextualization of Moral Work: An Analysis of Reproaches in 'Why'-Formats, in: Couper-Kuhlen E./Selting M. (Hrsg.), Prosody in Conversation: Interactional Studies, Cambridge, S. 271-302.

GÜNTHNER Susanne, 1997, The Contextualization of Affect in Reported Dialogues, in: Niemeier S./ Dirven R. (Hrsg.), The Language of Emotions, Amsterdam, S. 247-276.

GÜNTHNER Susanne, 2000, Vorwurfsaktivitäten in der Alltagsinteraktion. Grammatische, prosodische, rhetorisch-stilistische und interaktive Verfahren bei der Konstitution kommunikativer Muster und Gattungen, Tübingen.

GUTENBERG Norbert, 1998, Einzelstudien zu Sprechwissenschaft und Sprecherziehung. Arbeiten in Teilfeldern, Göppingen.

GRUNDLER Elke, 2011, Kompetent argumentieren. Ein gesprächsanalytisch fundiertes Modell, Tübingen.

HAUSENDORF Heiko / QUASTHOFF Uta, 2005, Sprachentwicklung und Interaktion. Eine linguistische Studie zum Erwerb von Diskursfähigkeiten, Radolfzell.

HAUSER Stefan / LuginBÜHL Martin, 2015, Aushandlung von Angemessenheit in Entscheidungsdiskussionen von Schulkindern, in: Aptum - Zeitschrift für Sprachkritik und Sprachkultur 11(2), S. $180-189$.

HeLler Vivien, 2012, Kommunikative Erfahrungen von Kindern in Familie und Unterricht. Passungen und Divergenzen, Tübingen.

HirschFELD Ursula / Neuber Baldur, 2010, Prosodie im Fremdsprachenunterricht Deutsch. Ein Überblick über Terminologie, Merkmale und Funktionen, in: Deutsch als Fremdsprache 1, S. $10-16$.

HIRSCHFELD Ursula / STOCK Eberhard, 2013, Phonologische Grundlagen des Deutschen, in: Bose I./ Hirschfeld U./Neuber B./Stock E. (Hrsg.), Einführung in die Sprechwissenschaft. Phonetik, Rhetorik, Sprechkunst, Tübingen, S. 27-47.

House Jill, 2006, Constructing a Context with Intonation, in: Journal of Pragmatics 38(10), S. $1542-1558$.

House Jill, 2009, Prosody and Context Selection: A Procedural Approach, in: Barth-Weingarten D./ Wichmann A./Dehé N. (Hrsg.), Where Prosody Meets Pragmatics, Bingley, S. 129-142. 
KAIMAKI Marianna, 2011, Sequentially Determined Function of Pitch Contours: The Case of English News Receipts, in: York Papers in Linguistics 2(2), S. 49-73, http://www.york.ac.uk/ language/ypl/yp12issue11/YPL11_03_Kaimaki.pdf.

KERN Friederike, 2010, Speaking Dramatically: The Prosody of Live Radio Commentary of Football Matches, in: Barth-Weingarten D./Reber E./Selting M. (Hrsg.), Prosody in Interaction, Amsterdam, S. 217-238.

KOMOR Anna 2010, Miteinander kommunizieren - Kinder unter sich. Eine empirische diskursanalytische Untersuchung zur Ausbildung kindlicher Kommunikationsfähigkeit, Münster/New York/München.

KOPPERSCHMIDT Josef, 2000, Argumentationstheorie zur Einführung, Hamburg.

KRECH Eva-Maria / RICHTER Günther / StOck Eberhard / SutTNER Jutta, 1991, Sprechwirkung. Grundfragen, Methoden und Ergebnisse ihrer Erforschung, Berlin.

KRELLE Michael, 2014, Mündliches Argumentieren in leistungsorientierter Perspektive. Eine empirische Analyse von Unterrichtsdiskussionen in der neunten Jahrgangsstufe, Hohengehren.

KuPETZ Maxi, 2014, ,Mitfühlend sprechen ‘ - Zur Rolle der Prosodie in Empathiedarstellungen, in: Barth-Weingarten D./Szczepek Reed B. (Hrsg.), Prosodie und Phonetik in der Interaktion Prosody and Phonetics in Interaction, Mannheim, S. 87-114.

KuRTENBACH Stephanie / BOSE Ines (Hrsg.), 2013, Gespräche zwischen Erzieherinnen und Kindern: Beobachtung, Analyse, Förderung, Frankfurt a. M.

LeITÃo Selma, 2010, Argumentação e cognição. Desenvolvimento de condutas proto-argumentativas (Projeto de Pesquisa/2010). Programa de Pós Graduação em Psicologia Cognitiva, Universidade Federal de Pernambuco, Recife, PE.

MoREK Miriam, 2012, Kinder erklären - Interaktionen in Familie und Unterricht im Vergleich, Tübingen.

MOREK Miriam, 2015a, Dissensbearbeitung unter Gleichaltrigen - (k)ein Kontext für den Erwerb argumentativer Gesprächsfähigkeiten?, in: Arendt B./Heller V./Krah A. (Hrsg.), Themenheft «Kinder argumentieren. Interaktive Erwerbskontexte und -mechanismen», in: Mitteilungen des Deutschen Germanistenverbandes, S. 34-46.

MoreK Miriam, 2015b, Show That You Know - Explanations, Interactional Identities and Epistemic Stance-taking in Family-talk and Peer-talk, in: Linguistics and Education 31, S. 238 - 259.

MÜLler Anja, 1999, Die Macht der Stimme. Die Stimme als rhetorischer Wirkungsfaktor: Zur persuasiven Funktion und Wirkung der Prosodie, Bad Iburg.

MÜLLER Frank E.,1996, Affiliating and Disaffiliating with Continuers: Prosodic Aspects of Recipiency, in: Couper-Kuhlen E./Selting M. (Hrsg.), Prosody in Conversation: Interactional Studies, Cambridge, S. 131-170.

NeUBER Baldur, 2001, Prosodische Form-Funktions-Relationen. Überlegungen zur Wahrnehmung und Interpretation der ,Musik' des Sprechens, in: Deutsch als Fremdsprache 2, S. 99-103.

NeUber Baldur, 2002, Prosodische Form in Funktion. Leistungen der Suprasegmentalia für das Verstehen, Behalten und die Bedeutungs(re)konstruktion, Frankfurt a. M.

Ogden Richard, 2006, Phonetics and Social Action in Agreements and Disagreements, in: Journal of Pragmatics 38, S. 1752-1775.

Piaget Jean, 1959, The Language and Thought of the Child (3. A), London.

QuASTHOFF Uta, 2009, Entwicklung der mündlichen Kommunikationskompetenz, in: Becker-Mrotzek M. (Hrsg.), Unterrichtskommunikation und Gesprächsdidaktik. Teilband Mündlichkeit in der Handbuchreihe Deutschunterricht in Theorie und Praxis, Baltmannsweiler, S. 84-100.

QUASTHOFF Uta / KRAH Antje, 2012, Familiale Kommunikation als Spracherwerbsressource: Das Beispiel argumentativer Kompetenzen, in: Neuland E. (Hrsg.), Sprache der Generationen, Mannheim, S. $115-132$.

REBER Elisabeth, 2009, Zur Affektivität in englischen Alltagsgesprächen, in: Buss M./Habscheid S./ Jautz S./Liedtke F./Schneider J. (Hrsg.), Theatralität des sprachlichen Handelns. Eine Metaphorik zwischen Linguistik und Kulturwissenschaften, München, S. 193-215. 
SACHS Jacqueline, 1983, Talking about the There and Then: The Emergence of Displaced Reference in Parent-Child Discourse, in: Nelson K. (Hrsg.), Children's Language 4, New York, S. 1-28.

SACKS Harvey, 1967/95, Lectures on Conversation, Oxford/Cambridge.

SCHILCHER Anita, 2000, Was brauchen wir auf einer einsamen Insel?, in: Praxis Deutsch 160 (Themenheft Argumentieren), S. 26-29.

SCHWARZE Cordula, 2010, Formen und Funktionen von Topoi im Gespräch. Eine empirische Untersuchung am Schnittpunkt von Argumentationsforschung, Gesprächsanalyse und Sprechwissenschaft, Frankfurt a. M.

SCHWITALLA Johannes, 1993, Über einige Weisen des gemeinsamen Sprechens. Ein Beitrag zur Theorie der Beteiligungsrollen im Gespräch, Zeitschrift für Sprachwissenschaft 11(1), S. 68-98.

Selting Margret, 1996, Prosody as an Activity-type Distinctive Cue in Conversation: The Case of So-called 'Astonished' Questions in Repair Initiation, in: Couper-Kuhlen E./Selting M. (Hrsg.), Prosody in Conversation: Interactional Studies, Cambridge, S. 231-270.

Selting Margret / Auer Peter / Barth-Weingarten Dagmar / Bergmann Jörg / Bergmann Pia / BIRKNER Karin / COUPER-KuHLEn Elizabeth / DEPPERMAnN Arnulf / Gilles Peter / GÜNTHNER Susanne / HARTUng Martin / Kern Friederike / Mertzlufft Christine / MeYer Christian / MoreK Miriam / Oberzaucher Frank / Peters Jörg / Quasthoff Uta / SchÜtTE Georg / StukenBrock Anja / Uhmann Susanne, 2009, Gesprächsanalytisches Transkriptionssystem 2 (GAT 2), in: Gesprächsforschung - Online-Zeitschrift zur verbalen Interaktion 10, S. $353-402$.

Selting Margret, 2013, Verbal, Vocal, and Visual Practices in Conversational Interaction, in: Müller C./Cienki A.J./Fricke E./Ladewig S.H./ McNeill D./Tessendorf S. (Hrsg.), Body - Language - Communication. An International Handbook on Multimodality in Human Interaction, Handbücher zur Sprach- und Kommunikationswissenschaft 38(1), Berlin, S. 589 - 609.

SIDNELL Jack, 2010, Questioning Repeats in the Talk of Four-Year-Old Children, in: Gardener H./ Forrester M. (Hrsg.), Analysing Interactions in Childhood, S. 103-127.

SPRANZ-FogASY Thomas, 2003, Alles Argumentieren oder was? Zur Konstitution von Argumentationen in Gesprächen, in: Deppermann A./Hartung, M. (Hrsg.), Argumentationen in Gesprächen, Tübingen, S. 27-39.

SPRANZ-FogASY Thomas, 2005, Argumentation als alltagsweltliche Kommunikationsideologie, in: Deutsche Sprache 2/05, S. 141-156.

STEIN Nancy L. / AlBRo Elizabeth R., 2011, The Origins and Nature of Arguments: Studies in Conflict Understanding, Emotion, and Negotiation, in: Discourse Processes 32(2\&3), S. 113-133.

STUDE Juliane, 2014, The Acquisition of Discourse Competence: Evidence from Preschoolers' Peer Talk, in: Learning, Culture and Social Interaction 3/2, S. 11-120.

SzCZEPEK Beatrice, 2000a, Formal Aspects of Collaborative Productions in English Conversation, in: InLiSt: Interaction and Linguistic Structures 17.

SzCZEPEK Beatrice, 2000b, Functional Aspects of Collaborative Productions in English Conversation, in: InLiSt: Interaction and Linguistic Structures 21.

SZCZEPEK Reed Beatrice, 2004, Turn-final Intonation in English, in: Couper-Kuhlen E./Ford C.E. (Hrsg.), Sound Patterns in Interaction, Amsterdam, S. 97-119.

SZCZEPEK Reed Beatrice, 2006, Prosodic Orientation in English Conversation, Basingstoke.

SzCZEPEK Reed Beatrice, 2012, Beyond the Particular: Prosody and the Coordination of Actions, in: Language and Speech 55(1), S. $13-34$.

TARPLEE Clare, 1996, Working on Young Children's Utterances: Prosodic Aspects of Repetition during Picture Labeling, in: Couper-Kuhlen E./Selting M. (Hrsg.), Prosody in Conversation: Interactional Studies, Cambridge, S. 406-435.

Tomasello Michael, 2003, Constructing a Language. A Usage-based Theory of Language Acquisition, Cambridge.

TYKKYLÄINEN Tuula, 2010, Child-initiated Repair in Task Interactions, in: Gardener H./Forrester M. (Hrsg.), Analysing Interactions in Childhood, S. 227-248. 
VAn EEMEREn Frans H. / GRoOTEndorst Rob, 2004, A Systematic Theory of Argumentation. The Pragma-dialectical Approach, Cambridge.

VASCONCELOS Angelina Nunes de, 2013, Argumentação desenvolvimento cognitivo. Emergência e estabilização de condutas protoargumentativas. Dissertation, Universidade Federal de Pernambuco, Recife, PE, http://repositorio.ufpe.br/bitstream/handle/123456789/10172/ Dissertação\%20Angelina\%20Nunes\%20de\%20Vasconcelos.pdf?sequence=1\&isAllowed=y.

VASCONCElos Angelina Nunes de / SCARPA Ester / DodANE Christelle, 2015, Emergence de la prosodie et de l'opposition. Le développement de la proto-argumentation. Präsentation an der Konferenz ARGAGE 2015 - Argumentation \& Language, 09.09.2015, Université de Lausanne.

VoGT Rüdiger, 2002, Im Deutschunterricht diskutieren. Zur Linguistik und Didaktik einer kommunikativen Praktik, Tübingen.

VyGOTSKY Lev S., 1978, Mind in Society, Cambridge.

WeLls Bill / CORRIN Juliette, 2004, Prosodic Resources, Turn-taking and Overlap in Children's Talk-in-Interaction, in: Couper-Kuhlen E./Ford C.E. (Hrsg.), Sound Patterns in Interaction, Amsterdam, S. $119-144$.

Wells Bill, 2010, Tonal Repetition and Tonal Contrast in English Carer-child Interaction, in: Barth-Weingarten D./Reber E./Selting M. (Hrsg.), Prosody in Interaction, Amsterdam, S. $243-262$.

ZADUNAISKY EHRLICH Sara / BLUM-KulKa Shoshana, 2014, "Now I said Danny becomes Danny again". A Multifaceted View of Kindergarten Children's Peer Argumentative Discourse, in: Cekaite A./Blum-Kulka S./Grover W./Teubal E. (Hrsg.), Cambridge/New York, S. 23-41. 